Reprod. Nutr. Dévelop., 1982, 22 (6), 999-1012.

\title{
La segmentation du canal épididymaire chez la souris : étude ultrastructurale
}

Lydia SORANZO (1), J.-P. DADOUNE, Marcelle-Anne FAIN-MAUREL $\left({ }^{*}\right)$

Laboratoire d'Histologie, Embryologie et Cytogénétique,

Faculté de Médecine Broussais Hôte/-Dieu

(*) Laboratoire de Biologie cellulaire, Université René Descartes, 45, rue des Saints-Pères, 75006 Paris, France.

Summary. Segmentation of the epididymal duct in mouse : an ultrastructural study.

The cytology of epithelial cells of the mouse epididymis was studied under light and electron microscopy. Four principal areas - the initial segment, the head, the body and the tail - were distinguished according to the morphological features of the main cells. Transitional areas separating these segments have been described also; one of the segments, intermediate between the head and the body, was longer. Five other cell types and their sites were noted : " apical " cells, corresponding to the "narrow " cells of Sun and Flickinger (1980), were located in the initial to the intermediate segment; clear cells were scattered in the epithelium from the intermediate segment to the tail ; basal cells were sporadically distributed along the entire duct. Halo and dark cells were observed also.

Les travaux les plus récents effectués chez les mammifères ne permettent pas d'établir une correspondance exacte entre les trois portions anatomiques (tête, corps, queue) du canal épididymaire et la structure microscopique (Hamilton, 1975 ; Ramos et Dym, 1977 ; Flickinger et al., 1978 ; Hoffer et Greenberg, 1978 ; Jones et al., 1979 ; Nicander et Ploen, 1979 ; Tingari et Moniem, 1979). De plus, la nature et la localisation des différents types cellulaires répartis entre les cellules principales de l'épithélium varient selon les espèces (Hamilton, 1975 ; Ramos et Dym, 1977 ; Flickinger et al., 1978 ; Sun et Flickinger, 1980).

Afin d'établir la correspondance entre les caractères cytologiques des différents types cellulaires et les portions histologiques de l'épididyme, une étude ultrastructurale systématique a été réalisée sur la longueur du canal chez la souris, espèce pour laquelle les données histologiques demeurent fragmentaires (Benoit, 1926 ; Allen et Slater, 1957 ; Barron et Mac Enery, 1966 ; Hamilton, 1972 ; Pavlok, 1974).

\section{Matériel et méthodes.}

Des souris mâles âgées de 2 mois et pesant environ $35 \mathrm{~g}$ ont été sacrifiées par perfusion intracardiaque de 2-fixateurs distribués successivement à l'aide

(1) Adresse actuelle : Laboratoire d'Anatomie pathologique, Hôpital Tenon, 4, rue de la Chine, 75970 Paris 20, France. 
d'un robinet à 2 voies : paraformaldéhyde à 3 p. 100 dans le tampon collidine $S$ $(0,2 \mathrm{M} ; \mathrm{pH}=7,2 ; 3 \mathrm{~min})$, puis glutaraldéhyde 1,6 p. 100 dans le même tampon $(5 \mathrm{~min})$.

L'épididyme a été prélevé et immergé dans le dernier fixateur pendant $1 \mathrm{~h}$. II a été disséqué dans le tampon et déroulé au niveau de la queue sous la loupe binoculaire. Des 5 cônes efférents issus du hile testiculaire, un seul s'abouche directement dans le segment initial, portion la plus antérieure de la tête anatomique, elle-même divisée par des cloisons conjonctives incomplètes. La tête est ainsi formée de 8 lobules discoïdaux empilés les uns sur les autres (fig. 1).

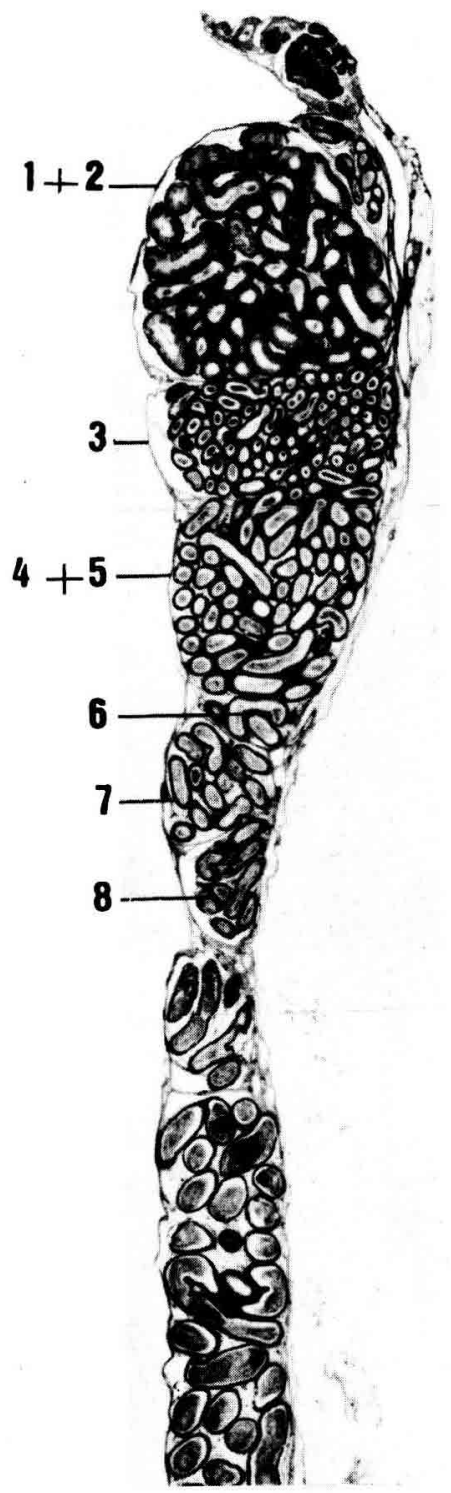

FIG. 1. - Section longitudinale de la tête anatomique divisée en 8 lobules discoïdaux séparés par des cloisons conjonctives incomplètes.

Segmentation histologique : - le segment initial occupe les lobules 1 et 2 ; - le segment de la tête histologique les lobules 3 et 4 ; - le segment intermédiaire les lobules 5 et 6 ; - la région du corps histologique commence dès le lobule 7 .

Coloration hémalun-éosine. Montage de 3 photos G $\times 15$. 
Des prélèvements échelonnés ont été effectués. Chaque lobule de la tête est isolé, numéroté ainsi que les portions proximale, moyenne et distale du corps et de la queue en se référant à une étude histologique préliminaire sur coupes longitudinales sériées du canal fixé dans le Bouin et inclus en paraffine (Soranzo, 1979).

Les échantillons ont été post-fixés dans l'acide osmique à 2 p. 100, déshydratés et inclus dans l'Epon. Après repérage sur coupes semi-fines, les coupes ultra-fines correspondantes, contrastées par l'acétate d'uranyle et le citrate de plomb ont été examinées au microscope électronique Siemens Elmiskop 101.

\section{Résultats.}

Particularités cytologiques des cellules principales des 4 segments histologiques.

Les caractéristiques cytologiques des cellules principales de l'épithélium du segment initial, du corps et de la queue de l'épididyme correspondent, pour l'essentiel, à la description fournie par Hamilton (1975) chez le rat. Toutefois, il faut noter quelques particularités chez la souris (PI. I).

Dans les cellules principales du segment initial (lobules 1 et 2), comme chez les autres espèces décrites, le réticulum endoplasmique vésiculaire apparaît très pauvre en ribosomes sur coupes ultra-fines dans la région supranucléaire (PI. II, fig. A), granulaire et sacculaire dans la région infranucléaire où il constitue une corbeille de citernes empilées (PI. II, fig. B). En outre, de nombreux corps résiduels sphériques et pseudo-lamellaires avoisinent avec les saccules golgiens très dilatés et l'ensemble constitue des particularités cytologiques des cellules principales de ce niveau (PI. II, fig. C). Les mitochondries effilées sont orientées parallèlement à l'axe de la cellule.

Par rapport au segment initial, l'aspect des cellules de la tête, (lobules 3-4) diffère sur les points suivants : la hauteur des cellules est réduite au détriment de la zone supragolgienne (PI. 1, fig. B ; PI. II, fig. D), des vésicules à contenu floculant sont observées entre les stéréocils ; les mitochondries plutôt globuleuses se regroupent, du moins dans la région basale, où elles côtoient les vésicules lisses.

Ces vésicules également disséminées dans tout le cytoplasme sont caractérisées par la variabilité de leur taille, l'épaisseur des membranes et, parfois, un nombre très réduit de ribosomes périphériques (PI. II, fig. F). Des peroxysomes révélés à la DAB (diamino-benzidine) selon la technique de Graham et Karnovsky (1966) sont également présents.

Dans ces deux segments, outre les complexes jonctionnels classiquement décrits, un aspect inhabituel de jonctions a été observé à la partie apicale des cellules principales. Les membranes plasmiques des cellules adjacentes délimitent un chenal rectiligne qui s'ouvre directement dans la lumière et qui est occupé par des trabécules transverses irrégulièrement espacés (PI. III, fig. A). 


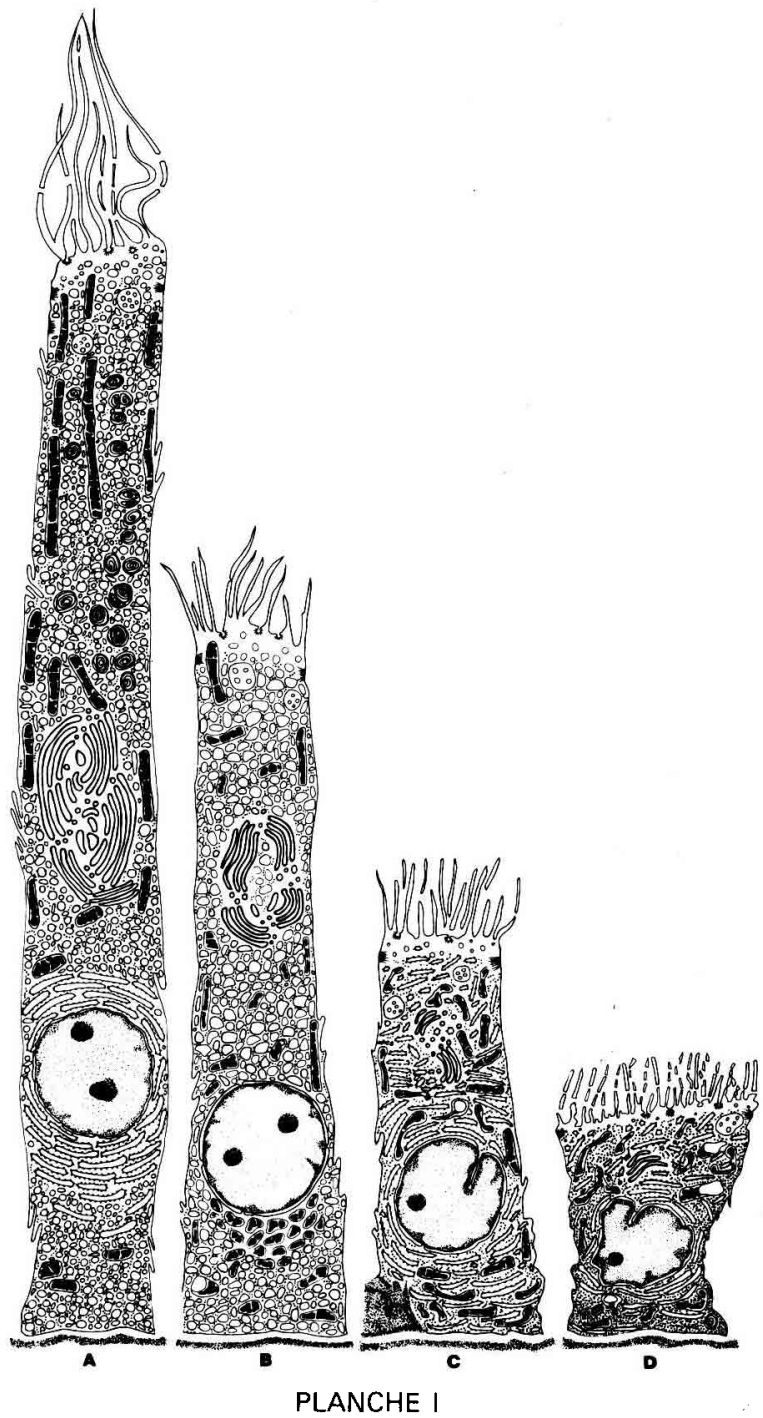

Représentation schématique chez la souris des cellules principales au niveau du segment initial (fig. A), de la tête (fig. B), du corps (fig. C) et de la queue (fig. D) qui mesurent respectivement $65 \mu \mathrm{m}, 35 \mu \mathrm{m}, 20 \mu \mathrm{m}$ et $10 \mu \mathrm{m}$ de hauteur totale.

\section{PLANCHE II}

FIG. A. - Segment initial, partie apicale $(\times 5200)$; FIG. B. - Segment initial, partie basale. Noter $(-1)$ l'empilement en corbeille des saccules de RE granulaire $(\times 5200)$; FIG. C. - Segment initial, aire golgienne $(\mathrm{g})$ riche en corps résiduels $(\mathrm{cr})(\times 18000)$; FIG. D. - Tête, partie apicale. Noter la situation de l'aire golgienne $(\mathrm{g})(\times 5200)$; FIG. E. - Tête, partie basale caractérisée par le groupement des mitochondries $(\rightarrow)(\times 5400)$; FIG. F. - Téte, zone apicale montrant le polymorphisme des vésicules. Noter, en regard des flèches, la présence de vésicules de réticulum pauvres en ribosomes, identiques à celles de la région supranucléaire du segment initial $(\times 18000)$. 
$7=r^{2}=2$

- c.

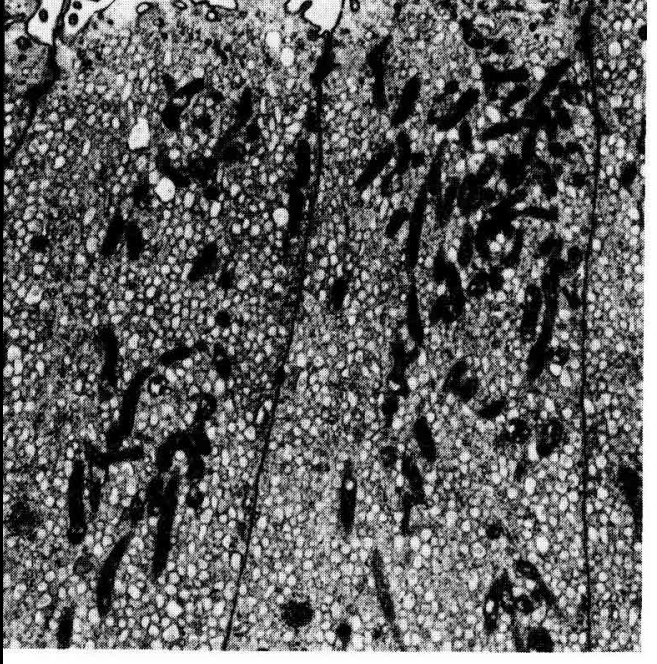

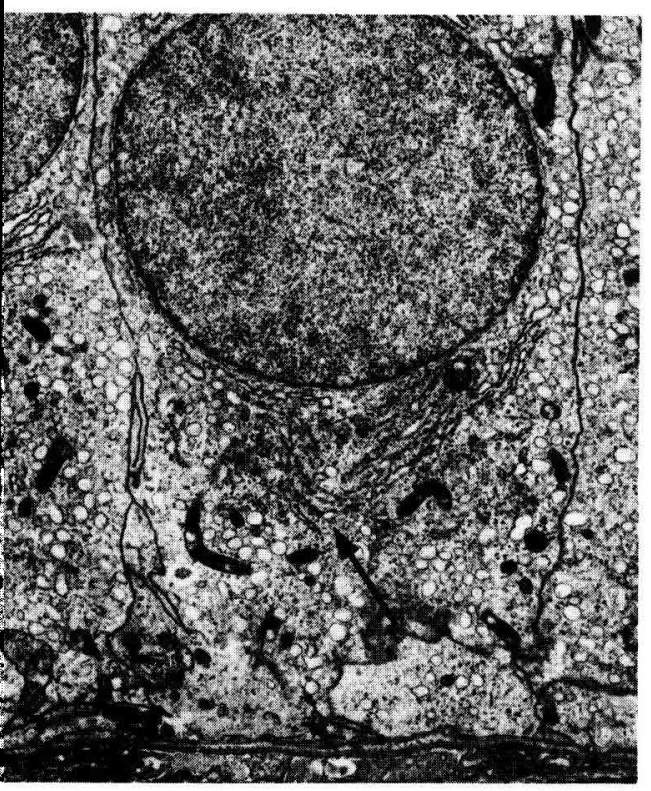

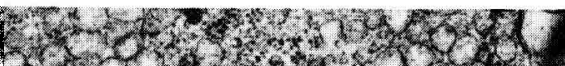

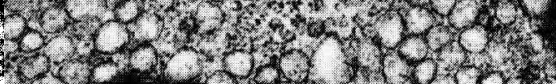

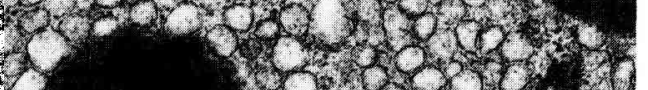

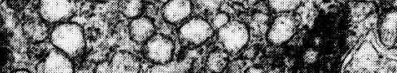

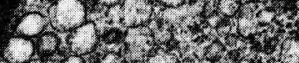

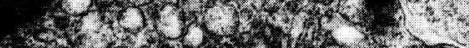

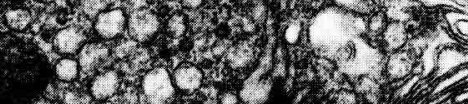
(1) 13 (a)

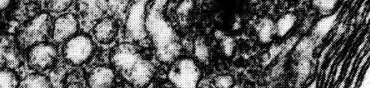

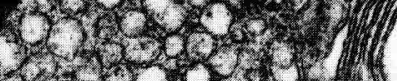

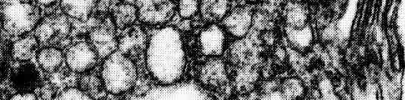

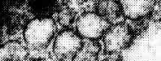

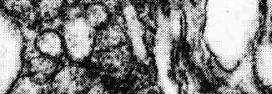

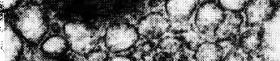

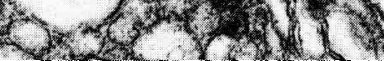

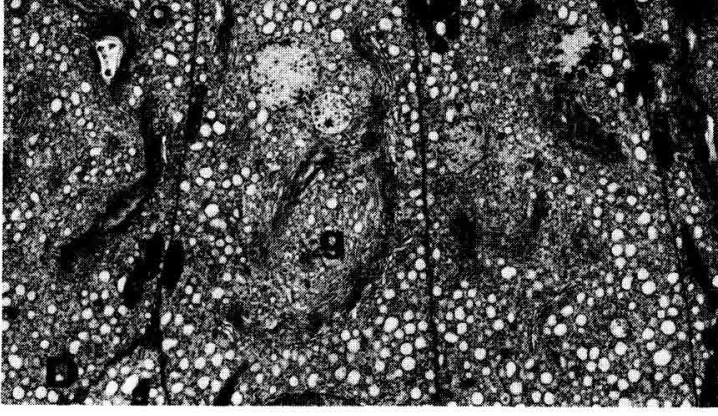

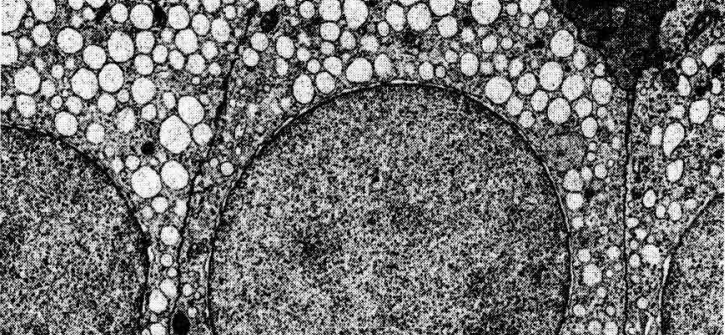
8050

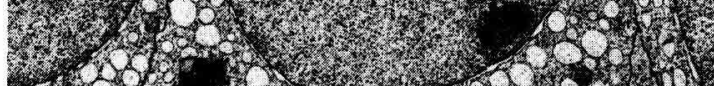

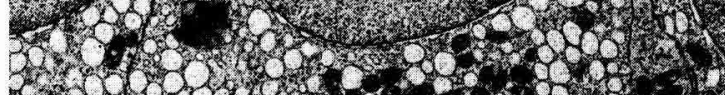

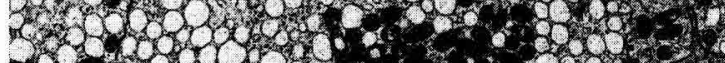

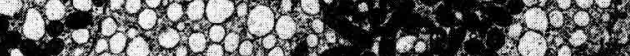

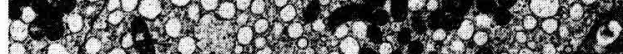

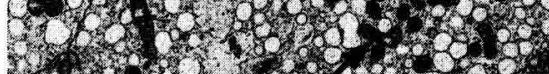

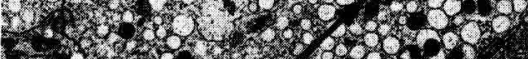

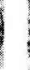

D. 6.5.

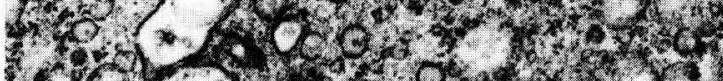

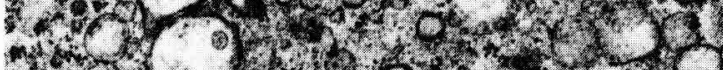
3.

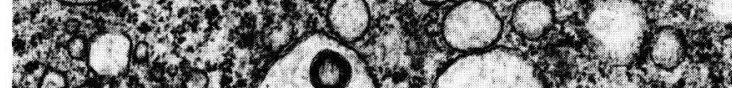

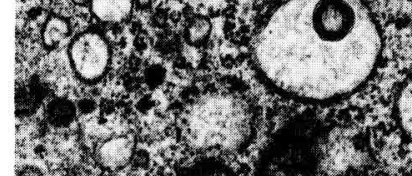


(3)

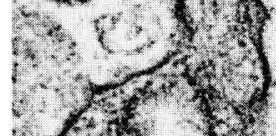

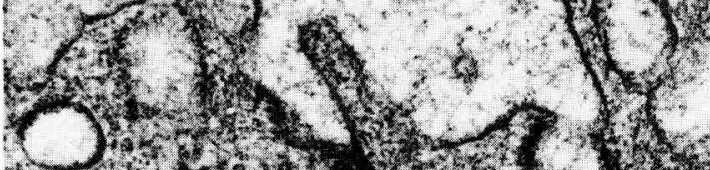

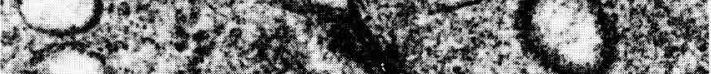

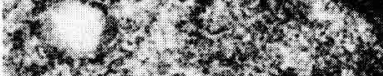
Mtr.

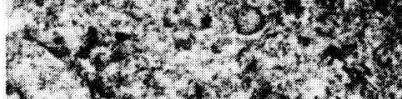

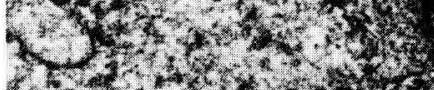

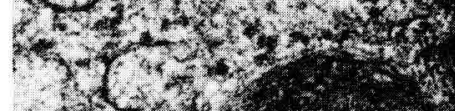

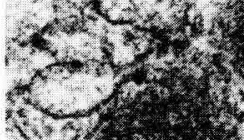

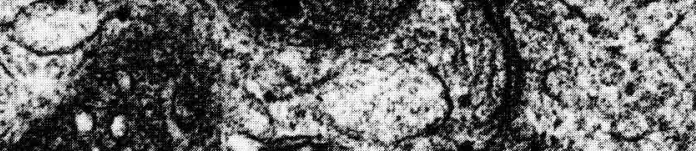

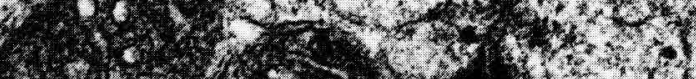

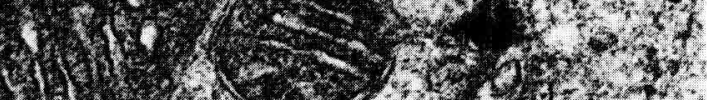

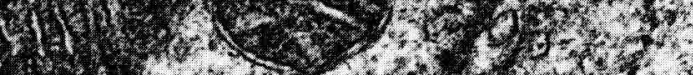
1.

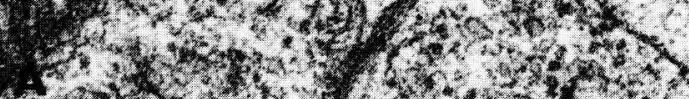

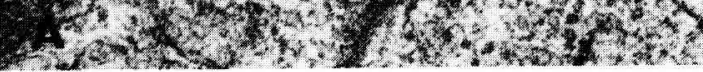

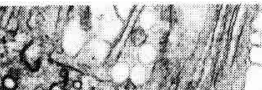

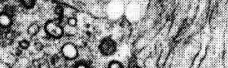

3.

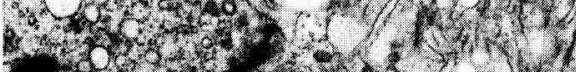

5.

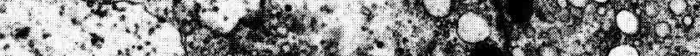

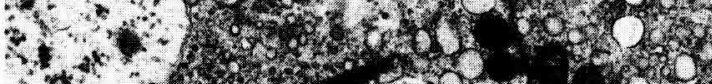

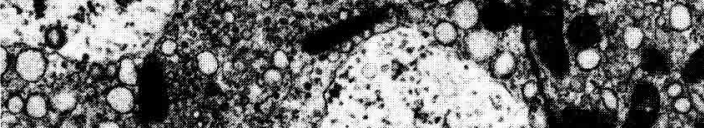

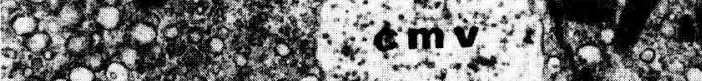

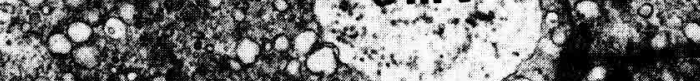

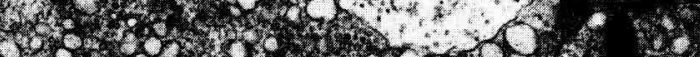
30.82 .

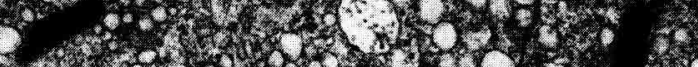

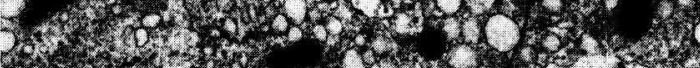

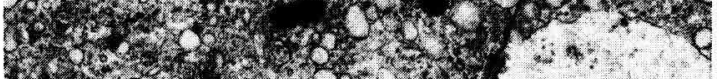

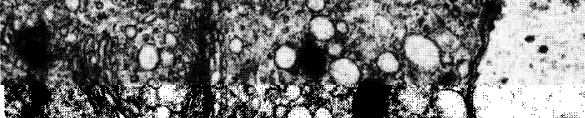

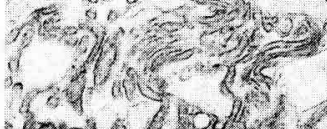

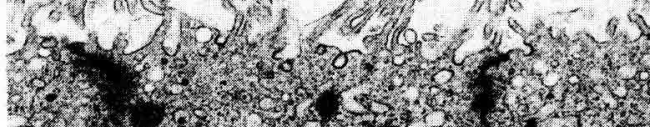

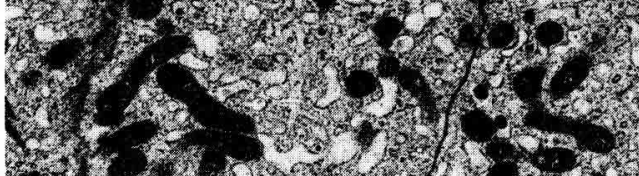
$1 / 4=-183$

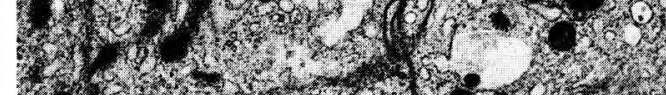

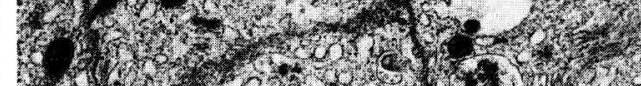

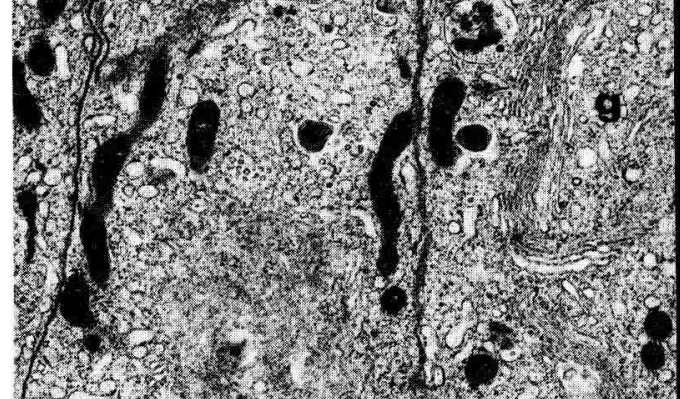
a

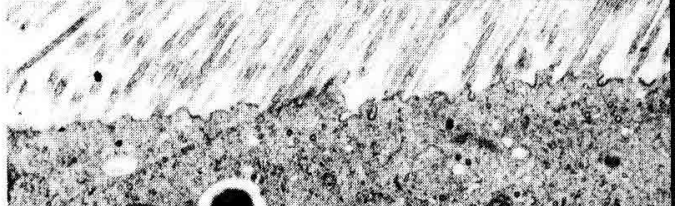

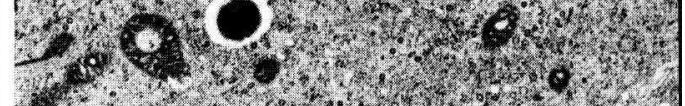

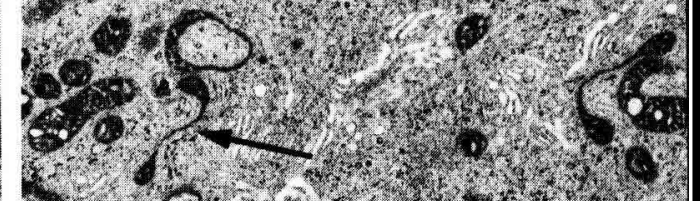

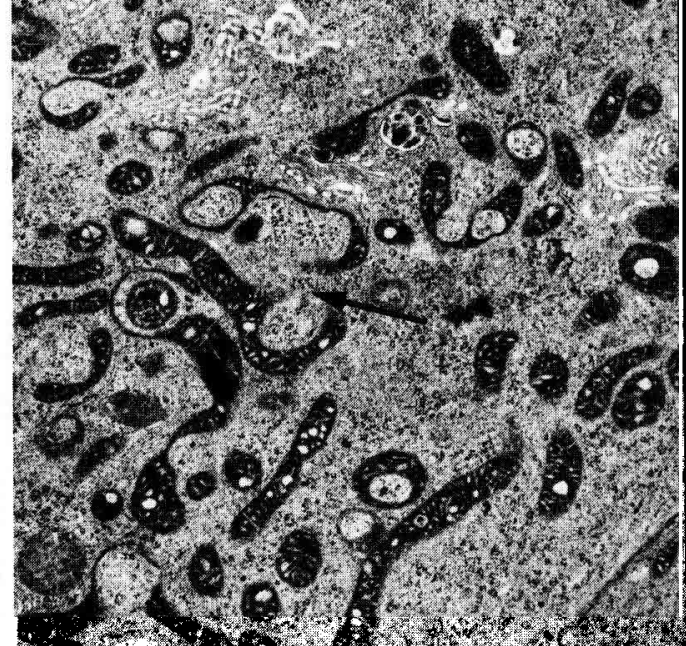


Dans la partie plus distale du canal (lobules 7, 8 corps et queue) la hauteur de I'épithélium diminue progressivement (PI. I, fig. $\mathrm{C}$ et D) ainsi que celle des stéréocils qui prennent l'aspect d'une bordure en brosse. La densité du cytoplasme augmente du fait de l'enrichissement en saccules de RE granulaire, longs, curvilignes ou parallèles dans le corps, puis courts et étroits dans la queue. L'appareil de Golgi est représenté par des dictyosomes plus dispersés. Les mitochondries dont la taille se réduit, présentent un aspect polymorphe dans le corps puis en haltère dans la queue. Les corps résiduels, très osmiophiles et hétérogènes sont particulièrement abondants dans ce dernier segment.

Les noyaux, pâles et ovoïdes dans la partie proximale du canal acquièrent ensuite un contour irrégulier puis indenté avec une chromatine en amas ou condensée à la périphérie dans la partie de la queue ( $P I .1$, fig. $A, B, C$ et D).

II ressort de l'étude ultrastructurale que les cellules principales présentent tout le long du canal épididymaire des critères morphologiques témoignant à la fois de la fonction d'absorption (stéréocils, pinocytose, corps multivésiculaires) et de la fonction de synthèse de protéines exportables (RE granulaire, dictyosomes) en dépit de l'absence constante du matériel élaboré.

Sur la base des caractéristiques cytologiques des cellules principales ainsi définies, des zones de transition ont été observées le long du canal épididymaire ainsi que 5 autres types cellulaires.

\section{Caractéristiques cytologiques des cellules principales des zones de transition.}

Deux caractéristiques ultrastructurales permettent de différencier les cellules de la zone de transition entre le segment initial et la tête : la diminution du nombre de vésicules du RE supranucléaire et surtout la richesse en volumineux corps multivésiculaires associés à de nombreuses vésicules de pinocytose (PI. III, fig. B).

Les cellules de la zone de transition entre la tête et le corps formant le segment intermédiaire (lobules 5 et 6) contiennent les organites qui s'apparentent par leur aspect à ceux de l'un ou l'autre de ces segments. Les dictyosomes sont groupés comme dans la tête. Les vésicules de réticulum endoplasmique sont moins nombreuses, peu dilatées, irrégulières dans leur forme et garnies de quelques ribosomes (PI. III, fig. C).

Les cellules de la zone de transition entre le corps et la queue sont identifiables par la transformation de la structure des mitochondries qui prennent une forme en haltère ou en cupule alors que le cytoplasme conserve toutes les caractéristiques ultrastructurales du segment sus-jacent (PI. III, fig. D).

\section{PLANCHE III}

FIG. A. - Nouveau type de complexe jonctionnel observé dans la partie proximale du canal $(\times 54000)$; FIG. B. - Zone de jonction entre le segment initial et la tête (cmv : corps multivésiculaire) ( $\times 7300)$; FIG. C. - Zone de jonction entre la tête et le corps (g : aire golgienne) $(\times 5300)$; FIG. D. - Zone de jonction entre le corps et la queue $(\rightarrow$ : mitochondries en haltère ou cupule) $(\times 5300)$. 
Au niveau de la queue distale ou zone pré-déférentielle, les cellules principales augmentent à nouveau de hauteur, et annoncent celles du canal déférent. On y note un cytoplasme dense et riche en REG où les tubules sont longs, les mitochondries plutôt allongées et le noyau clair avec de rares incisures (hauteur de l'épithélium $=30 \mathrm{~nm}$ ).

\section{Caractéristiques cytologiques des autres types cellulaires.}

De rares cellules étroites et denses, à noyau en position apicale sont disséminées dans le segment initial, la tête et le segment intermédiaire (PI. IV, fig. $A_{1}$ ). Au microscope électronique, elles présentent un aspect en calice avec une portion infranucléaire effilée qui se prolonge principalement au niveau de la zone intermédiaire par un pied élargi au contact de la lame basale. La membrane apicale émet des villosités courtes, épaisses et irrégulières. L'aire sous-jacente contient de petites vésicules lisses et pléomorphes, alors que le reste du cytoplasme est riche en REL développé en citernes tubulaires et vésiculaires compactes et denses. Les mitochondries forment deux amas respectivement situés dans la région supranucléaire et, accessoirement, dans la partie basale du pied de la cellule (PI. VI, fig. B).

Les cellules claires, prismatiques, localisées dès le segment intermédiaire et surtout dans la queue de l'épididyme sont identifiables en microscopie photonique par l'aspect clarifié de leur cytoplasme (PI. IV, fig. $A_{2}$ ). Sous la membrane plasmique apicale qui émet quelques digitations fines et irrégulières se remarquent de nombreuses vésicules polymorphes (PI. IV, fig. C). Ces cellules sont caractérisées par l'existence de deux compartiments : le premier, supranucléaire, est occupé essentiellement par des corps multivésiculaires de grande taille autour desquels se groupent de volumineuses mitochondries globuleuses se détachant d'un fond cytoplasmique dont l'aspect rappelle celui de la cellule apicale. Le second, périnucléaire et basal, contient de multiples corps sphériques de taille et de densité aux électrons variables ainsi que de longs saccules de RE granulaire orientés parallèlement à la lame basale. Des micro-peroxysomes ont été observés.

Les cellules basales, rares et refoulées contre la lame basale le long du canal épididymaire, présentent un noyau irrégulier, un cytoplasme réduit et pauvre en organites. Elles sont limitées par une membrane plasmique qui forme de nombreuses interdigitations avec celle des cellules contiguës (PI. IV, fig. D).

FIG. A. - Coupes semi-fines au niveau de la tête $\left(\mathrm{A}_{1}\right)$ où sont localisées les cellules apicales $(\rightarrow)$ et au niveau de la queue $\left(A_{2}\right)$ riche en cellules claires $(\rightarrow)(\times 400)$; FIG. B. - Montage montrant le corps cellulaire et le pied d'une cellule apicale ( $d^{\prime}$ narrow-cell ») ( $\left.\times 5300\right)$; FIG. C. - Montage montrant une cellule claire avec son compartiment apical contenant les corps multivésiculaires clairs (cmv) et son compartiment basal riche en corps denses (cd) $(\times 5300)$; FIG. D. Cellule basale $(\mathrm{cb})$ montrant les engrènements $(\rightarrow)$ de la membrane plasmique $(\times 5300)$. 


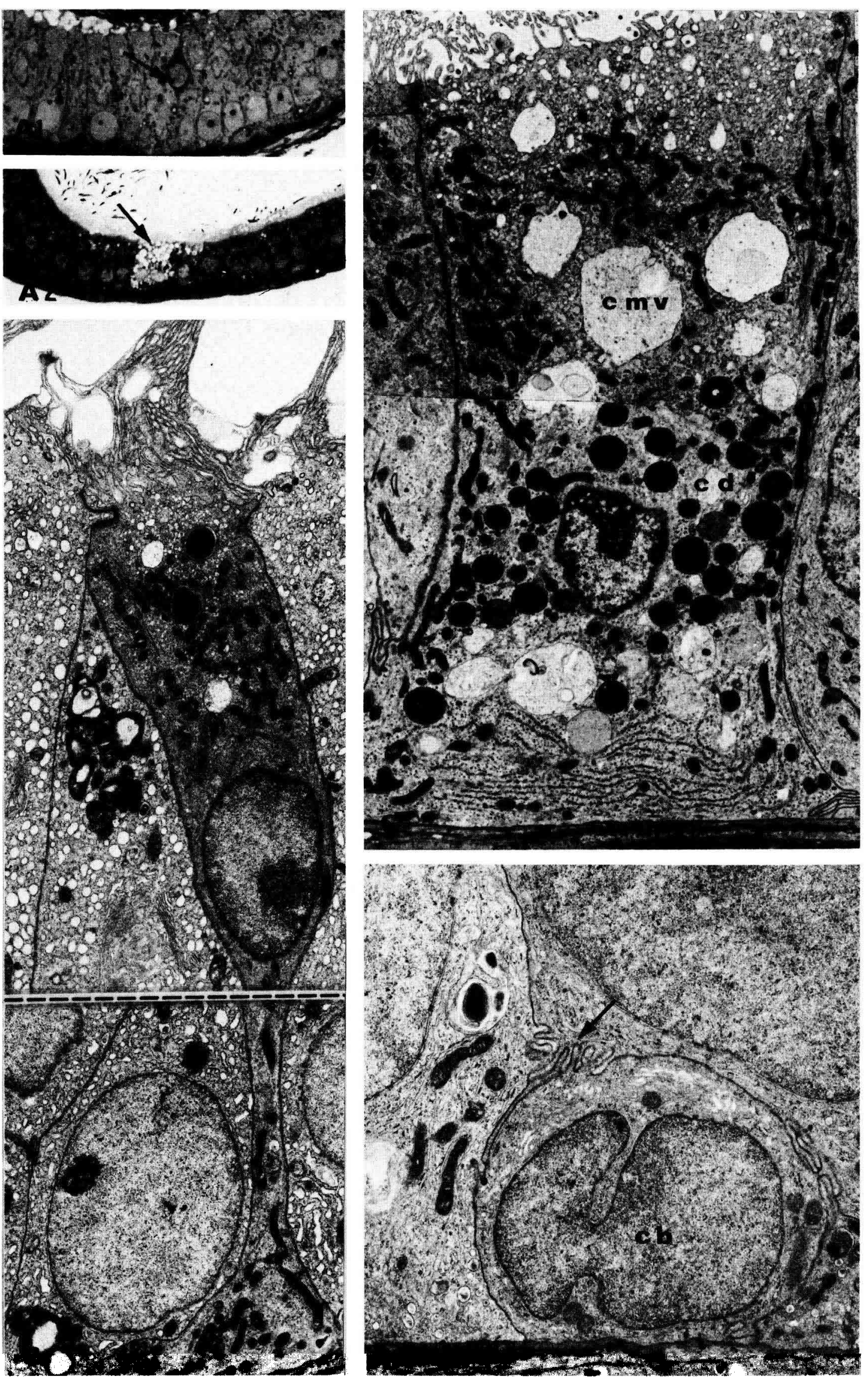


Quelques cellules arrondies, très semblables à des lymphocytes, sont trouvées dans la profondeur de l'épithélium essentiellement dans la partie initiale du canal.

Des cellules sombres sont observées à tous les niveaux avec la plus forte concentration dans la région de la queue où elles se regroupent par plages. Leur aspect évoque celui de cellules principales en dégénérescence.

\section{Discussion.}

Il apparaît que la nomenclature reposant sur la dénomination anatomique de l'épididyme en tête - corps - queue ne reflète nullement une segmentation histologique. Les différentes zones histologiques sont déterminées par le changement de structure des cellules principales et la localisation restreinte des « cellules apicales » et des cellules claires. Ni le groupe taxonomique auquel le mammifère appartient ni sa taille n'ont une répercussion sur le nombre de ces zones. En effet, 6 zones ont été distinguées chez le rat (Reid et Cleland, 1957), l'étalon, le bélier et le taureau (Nicander, 1958), 8 chez l'homme (Holstein, 1969), 7 chez le cobaye (Hoffer et Greenberg, 1978), 5 chez le chameau (Tingari et Moniem, 1979). Mais signalons que les résultats peuvent diverger selon leur obtention en microscopie optique (MO) ou électronique (ME). Chez le lapin, Nicander (1957) présente 8 zones en MO alors que Jones et al. (1979) en signalent 6 en ME.

Glover et Nicander (1971) ont proposé, sur la base de critères physiologiques et phylogéniques, de distinguer trois régions fonctionnelles de longueur variable selon l'espèce : le segment initial et le segment moyen qui assurent la maturation des spermatozoïdes, le segment terminal qui les stocke. Cependant, cette nomenclature ne rend pas compte de la diversité cellulaire de l'épithélium épididymaire. En second lieu, elle ne prend en considération que les fonctions par rapport aux gamètes et sous-estime considérablement l'éventualité de l'existence d'autres fonctions en rapport avec la variabilité ultrastructurale de ces cellules.

Effectivement, les cellules épithéliales épididymaires interviennent dans les variations de la composition du fluide le long du canal par leur activité mixte d'absorption et de sécrétion. La fonction de réabsorption est particulièrement évidente dans le segment initial du rat (Burgos, 1964) alors que chez la souris une étude morphométrique effectuée sur la tête, le corps et la queue a indiqué que le nombre et la répartition des corps multivésiculaires ne permettent pas de définir une région préférentielle en relation avec l'absorption (Vendrely et al., 1980). La synthèse et l'excrétion de protéines par les cellules principales ont été attestées chez la souris par plusieurs études autoradiographiques (Fain-Maurel et al., 1977 ; Kanka et Kopecny, 1977 ; Flickinger, 1979 ; Fain-Maurel et al., 1981). Après incorporation d'arginine, de leucine et de lysine tritiées, il apparaît qu'une partie des protéines élaborées au niveau de la tête, du corps et de la queue sont synthétisées selon le schéma habituel et excrétées rapidement (FainMaurel et al., 1981). D'autre part, diverses glycoprotéines épididymaires ont été mises en évidence par des techniques immuno-cytochimiques. Une glycoprotéine 
acide a été localisée dans les cellules épithéliales de la tête et du corps chez le rat (Lea et al., 1978) ainsi qu'un groupe de glycoprotéines spécifiques au niveau de la tête et du corps chez le hamster, le lapin (Moore, 1980) et le rat (Kohane et al., 1980). Enfin, le taux élevé de glycérylphosphorylcholine dans les cellules principales de la tête épididymaire permet de supposer l'intervention de ces cellules, au moins à ce niveau, dans le métabolisme de ce phosphoglycéride (Killian et Chapman, 1980). En dépit de la richesse en réticulum endoplasmique lisse des cellules dans certaines espèces la synthèse de stéroïdes dans l'épithélium épididymaire n'a été démontrée qu'in vitro (Hamilton et Fawcett, 1970). En revanche, la captation de la testostérone par l'épididyme et sa conversion en $5 \alpha$ dihydrotestostérone ont été mentionnées, en particulier au niveau de la tête (de Larminat et al., 1980).

Chez la souris, quatre portions histologiques principales peuvent être définies le long du canal sur la base des caractères ultrastructuraux des cellules (PI. I). Le segment initial est caractérisé par la richesse des cellules principales en $R E$ sacculaire et granulaire en position infranucléaire, vésiculaire et pauvre en ribosomes au-dessus du noyau. La tête se différencie du segment précédent par la présence d'un amas de mitochondries dans la partie basale des cellules. Le corps se reconnaît à l'augmentation de la densité du cytoplasme due à l'abondance de saccules de RE granulaire, la queue par l'aspect des mitochondries en haltères et les nombreux corps résiduels. Toutefois, le passage d'un segment à l'autre ne se fait pas de manière abrupte mais par la transformation progressive des caractéristiques cytologiques des cellules principales. Chez le lapin, il existe entre le segment initial et la tête (Nicander, 1981), une zone de transition bien définie dans laquelle s'accumulent les spermatozoïdes et dont les cellules principales sont particulièrement riches en corps multivésiculaires. Chez la souris, cette zone, dont les limites sont peu précises, également riche en corps multivésiculaires, pourrait représenter une entité fonctionnelle. La zone du segment intermédiaire quant à elle est remarquable par sa longueur (lobules 5 et 6 ) et l'aspect de son réticulum endoplasmique à mi-chemin entre le REG et le REL.

Chez la souris, 5 autres types cellulaires ont été observés : les cellules apicales, les cellules claires, les cellules basales, les cellules sombres et les cellules en halo. Ces dernières qui ont été retrouvées chez le singe (Ramos et Dym, 1977), le rat (Hoffer et al., 1973) et le hamster (Flickinger et al., 1978), sont considérées comme des lymphocytes intraépithéliaux. Les cellules apicales ont été décrites chez le singe (Ramos et Dym, 1977), les cellules claires chez le hamster (Flickinger et al., 1978). Toutes deux sont inconstantes selon les espèces.

"Les cellules apicales " sont, cheż la souris, localisées dans le segment initial, le segment histologique de la tête et le segment intermédiaire. Nos observations nous conduisent, en fait, à assimiler ces cellules aux " narrow cells ", décrites chez le rat adulte (Sun et Flickinger, 1980), qui diffèrent notablement de la variété de cellule principale à noyau apical désignée par Reid et Cleland (1957) sous le nom de cellules apicales. Leur capacité d'ingérer par endocytose de la peroxydase exogène est en faveur de leur intervention dans la réabsorption (Moore et Bedford, 1979). 
En ce qui concerne les cellules claires localisées à partir du segment intermédiaire jusqu'à la queue, plusieurs critères morpho-fonctionnels permettent également de leur attribuer une fonction de réabsorption par leur équipement en corps multivésiculaires et en lysosomes, leur pouvoir d'incorporer par pinocytose la peroxydase exogène (Moore et Bedford, 1979) et la glycoprotéine acide épididymaire élaborée dans les cellules principales des segments sus-jacents (Lea et al., 1978).

Chez la souris, comme chez les autres espèces, la signification des cellules basales reste à élucider. L'extrême rareté des mitoses, observées généralement dans la portion moyenne de l'épithélium, ne permet pas de leur attribuer un rôle déterminant dans le renouvellement cellulaire. II faut noter que, chez le rat, au niveau de la tête, un taux significatif de glycérylphosphorylcholine a été mis en évidence dans ces éléments (Killian et Chapman, 1980).

En conclusion, l'étude ultrastructurale systématique du canal épididymaire chez la souris a permis de mettre en évidence quatre segments histologiques principaux identifiables par les caractéristiques des cellules principales. En particulier, les cellules du segment initial et de la tête sont, chez cette espèce, tout à fait distinctes. Les zones de transition ont pu être observées, notamment, l'une remarquable par l'abondance des corps multivésiculaires, l'autre par sa longueur. Les fonctions précises de chacune de ces zones principales ou de transition méritent une exploration. Les très grandes variations des cellules principales de l'épididyme rendent difficile d'établir des corrélations structurales et fonctionnelles interspécifiques. C'est pourquoi toute taxonomie, qu'elle soit histologique ou morphofonctionnelle, doit être précisée avec soin lorsqu'elle sert de référence et devrait permettre une meilleure connaissance des fonctions épididymaires à différents niveaux. Toutes les variétés cellulaires décrites dans l'épididyme des mammifères sont représentées chez la souris, étant entendu qu'il convient désormais de réserver le terme de « narrow cells» aux cellules dites « apicales ».

Reçu en janvier 1982 Accepté en juillet 1982

\section{Références}

ALLEN J. M., SLATER J. J., 1957. A chemical and histochemical study of alkaline phosphatase and aliesterase in the epididymis of normal and castrate mice. Anat. Rec., 129, 255-273.

BARRON J., MAC ENERY W., 1966. The serial histology of the epididymis of the mouse. (Abstr.) Anat. Rec., 154, 2, 449.

BENOIT J., 1926. Recherches anatomiques, cytologiques et histophysiologiques sur les voies excrétrices du testicule, chez les mammifères. Arch. Anat. Histol. Embryol., 5, 173-412.

BURGOS M. H., 1964. Uptake of colloïdal particles by cells of the caput epididymis. Anat. Rec., 148, 517-525.

FAIN-MAUREL M. A., DADOUNE J. P., ALFONSI M. F., 1977. Modalités d'incorporation de l'arginine tritiée dans l'épithélium du canal épididymaire de souris. Biol. cell., 29, 7a. 
FAIN-MAUREL M. A., DADOUNE J. P., ALFONSI M. F., 1981. High resolution autoradiographic study of newly formed proteins in different segments of the epididymis after incorporation of tritiated amino acids. Arch. Androl., 6. 249-261.

FLICKINGER C. J., 1979. Synthesis, transport and secretion of protein in the initial segment of the mouse epididymis as studied by electron microscope radioautography. Biol. Reprod., 20, 1015-1030.

FLICKINGER C. J., HOWARDS S. S., ENGLISH H. F., 1978. Ultrastructural differences in efferent ducts and several regions of the epididymis of the hamster. Am. J. Anat, 152, 557586.

GLOVER T. D., NICANDER L., 1971. Some aspects of structure and function in the mammalian epididymis. J. Reprod. Fert., suppl., 13, 39-50.

GRAHAM R. C., KARNOVSKY M., 1966. The early states of absorption of injected horseradish peroxidase in the proximal tubule of mouse kidney. J. Histochem. cytochem., 14, 291-302.

HAMILTON D. W., 1972. The mammalian epididymis, 268-337. In BALIN H., GLASSER S., Biology of reproduction. Excerpta med. Found., Amsterdam, New-York.

HAMILTON D. W., 1975. Structure and function of the epithelium lining the ductuli efferentes, ductus epididymis, and ductus deferens in the rat. In Handbook of physiology, sect. 7 , vol. 5, 259-301 (Am. Physiol. Soc. Washington).

HAMILTON D. W., FAWCETT D. W., 1970. In vitro synthesis of cholesterol and testosterone from acetate by rat epididymis and vas deferens. Proc. Soc. exp. Biol. Med., 133, 693-695.

HOFFER A. P., GREENBERG J., 1978. The structure of the epididymis, efferent ductules and ductus deferens of the guinea-pig. A light microscope study. Anat. Rec., 190, 659-678.

HOFFER A. P., HAMILTON N. W., FAWCETT D. W., 1973. The ultrastructure of the principal cells and intraepithelial leucocytes in the initial segment of the rat epididymis. Anat. Rec., $175,169-201$.

HOLSTEIN A. F., 1969. Morphologische Studien am Nebenhoden des Menschen. Zwang/. Abh. Geb. Norm. Path. Anat., 20, 1-91.

JONES R., HAMILTON D. W., FAWCETT D. W., 1979. Morphology of the epithelium of the extratesticular rete testis, ductuli efferentes and ductus epididymis of the adult male rabbit. Am. J. Anat., 156, 376-400.

KANKA J., KOPEĆNY V., 1977. An autoradiographic study of macromolecular synthesis in the epithelium of the ductus epididymis in the mouse. I. DNA, RNA and protein. Biol. Reprod., 16, 421-427.

KILLIAN G. J., CHAPMAN D. A., 1980. Glycerylphosphorylcholine, sialic acid and protein in epithelial cells isolated from the rat caput epididymidis by elutriation. Biol. Reprod., 22, 846850.

KOHANE A. C., CAMEO M. S., PINEIRO L., GARBERI J. C., BLAQUIER J. A., 1980. Distribution and sites of production of specific proteins in the rat epididymis. Biol. Reprod., 23, 181-187.

LARMINAT M. A. de, HINRICHSEN M. J., SCORTICATI C., GHIRLANDA J. M., BLAQUIER J. A., CALANDRA R. S., 1980. Uptake and metabolism of androgen by the human epididymis in vitro. J. Reprod. Fertil., 54, 397-402.

LEA O. A., PETRUSZ P., FRENCH F. S., 1978. Purification and localization of acidic epididymal glycoprotein (AEG) : a sperm-coating protein secreted by the rat epididymis. Int. J. Androl., suppl., 2, 592-607.

MOORE H. D. M., 1980. Localization of specific glycoproteins secreted by the rabbit and hamster epididymis. Biol. Reprod., 22, 705-718.

MOORE H. D. M., BEDFORD J. M., 1979. The differential absorptive activity of epithelial cells of the rat epididymis before and after castration. Anat. Rec., 193, 313-328.

NICANDER L., 1957. On the regional histology and cytochemistry of the ductus epididymidis in rabbits. Acta morphol. neerl-scand., 1, 99-118.

NICANDER L., 1958. Studies on the regional histology and cytochemistry of the ductus epididymidis in stallions, rams and bulls. Acta morph. neerl.-scand., 1, 337-362.

NICANDER L., 1981. A cytologically specialized, highly androgen-dependent region in the epididymal head, related to structural maturation of spermatozoa. Int. J. Androl., suppl., 3. 66-67. 
NICANDER L., PLOEN L., 1979. Studies on regional fine structure and function in the rabbit epididymis. Int. J. Androl., 2, 463-481.

PAVLOK A., 1974. Development of the penetration activity of mouse epididymal spermatozoa in vivo and in vitro. J. Reprod. Fert., 36, 203-205.

RAMOS A. S. Jr, DYM M., 1977. Fine structure of the monkey epididymis. Am. J. Anat., 149, 501-532.

REID B. L., CLELAND K. W., 1957. The structure and function of the epididymis. 1. The histology of the rat epididymidis. Aust. J. Zool., 5, 223-246.

SORANZO L., 1979. Etude anatomique, histologique et ultrastructurale du canal épididymaire de la souris. Biol. cell., 35, 18a.

SUN E. L., FLICKINGER C. J., 1980. Morphological characteristics of cells with apical nuclei in the initial segment of the adult rat epididymis. Anat. Rec., 196, 285-293.

TINGARI M. D., MONIEM K. A., 1979. On the regional histology and histochemistry of the epididymis of the camel (Came/us dromedarius). J. Reprod. Fertil., 57, 11-20.

VENDRELY E., FAIN-MAUREL M. A., DADOUNE J. P., 1980. Apports de la morphométrie à l'étude ultrastructurale des cellules principales de l'épithélium épididymaire chez la souris adulte. Biol. cell., 38, 16a. 